\title{
OPTIMAL CFO CHANNEL ESTIMATION FOR ADAPTIVE RECEIVER DESIGN
}

\author{
Bakiya.A ${ }^{1}$, Kavyalakshmi.K ${ }^{2}$, Gayathri. ${ }^{3}$, Jeevitha.K ${ }^{4}$ \\ ${ }^{1}$ ECE, S.K.P Engineering college, Thiruvannamalai, Tamilnadu, India \\ ${ }^{2}$ ECE, S.K.P Engineering college, Thiruvannamalai, Tamilnadu, India \\ ${ }^{3}$ ECE, S.K.P Engineering college, Thiruvannamalai, Tamilnadu, India \\ ${ }^{4}$ ECE, S.K.P Engineering college, Thiruvannamalai, Tamilnadu, India
}

\begin{abstract}
An efficient of the inter carrier interference (ICI) cancellation in the adaptive receiver using orthogonal frequency division multiplexing system by two path conjugate transmission. At the receiver side the phase rotated conjugate cancellation (PRCC) concept is used and only one of the phase rotation is employed on the transmit path and two of the phase rotation is employed on the two of the receiver path. The main objective is to maximize the carrier-to-interference ratio and signal to noise ratio to achieve a high spectral efficiency using the fast least mean square algorithm to improve the bit error rate performance. The carrier frequency offset occurs in the mobile environment due to Doppler effects. Here the OFDM is used because it is highly sensitive to the carrier frequency offset.
\end{abstract}

Keywords: ICI cancellation, OFDM, two path conjugate transmission, PRCC, FLMS.

$* * *$

\section{INTRODUCTION}

OFDM provides a high rate of services and achieves a high spectral efficiency. It is sensitive to carrier frequency offset. It converts a high rate of input data streams into lower rate of data streams and transmitting them in the spectrally overlapped subcarriers. They are used in DVB, 3GPP LTE, wireless metropolitan area networks. The CFO problem that occurs due to the mismatch between the local oscillators at the transmitter and the receiver is highly sensitive to the OFDM. Orthogonal frequency-division multiplexing (OFDM) is a method of encoding digital data on multiple carrier frequencies. OFDM has developed into a popular scheme for wideband digital communication, whether wireless or over copper wires, used in application such as digital television and audio broadcasting, DSL internet access, wireless networks, and $4 \mathrm{G}$ mobile communications. OFDM is essentially identical to coded OFDM (COFDM) and discrete multi-tone modulation (DMT), and is a frequency-division multiplexing (FDM) scheme used as a digital multi-carrier modulation method. The primary advantage of OFDM over single-carrier schemes is its ability to cope with several channel condition without complex equalization filters. Channel equalization is simplified because OFDM may be viewed as using many slowly modulated narrowband signals rather than one rapidly modulated wideband signal. The low symbol rate makes the use of a guard interval between symbols affordable, making it possible to eliminate inter-symbol-interference (ISI) and utilize echoes and time-spreading to achieve a diversity gain that is signal-to-noise ratio improvement.

\section{BACKGROUND OF CC AND PRCC:}

The problem of the $\mathrm{CFO}$ can be mitigated by the synchronization of the carrier frequency at the transmitted and the received signals. Employ the pilot subcarriers for the ICI cancellation in fast fading multipath channels. Data symbol transmitted on to a group of subcarrier ICI induced from distinct carrier is mutually cancelled. The disadvantage is the spectral efficiency is reduced. The phase error generated limits the bit error rate performance. The two ICI self cancellations are the conjugate cancellation (CC) and the phase rotated conjugate cancellation (PRCC). In conjugate cancellation on the OFDM symbols is transmitted along the one path while its conjugate replicate is transmitted along two paths. After combining the received signal from the two paths ICI in one path is suppressed by the other path. In PRCC an artificial phase rotation the transmission of $\mathrm{CC}$ where the ICI induced from both the path can be mutual. The CFO changes with time the CC and PRCC does not provide a satisfactory performance even though they are time-variant channels. The receiver in the PRCC to determine the optimal phase rotation must feed the CFO back to the transmitter.

An adaptive receiver using two path conjugate transmission for efficient of the inter carrier interference cancellation in orthogonal frequency division multiplexing system. We employ two of the phase rotation on both the path of the receiver and one of the phase rotations is employed at the transmitter. By using block least mean-squared algorithm feeding back the CFO information can be eliminated. 
In the existing paper we developed a reduced complexity hybrid frequency/time-domain channel estimation algorithm which improves performance significantly under high Doppler with substantial complexity reductions. To further suppress ICI, we integrated this channel estimation algorithm with an ICI-mitigating pilot/data placement and joint channel/data detection schemes, and demonstrated further appreciable performance improvements even at a very high Doppler of $20 \%$. While we consider DVB-Has an application, the techniques presented in the existing paper can be applied to other mobile OFDM systems such as Wimax. The problem of synchronization occurs, symbol synchronization, frequency synchronization. The symbol synchronization is due to the timing errors and carrier phase noise. Frequency synchronization is due to the carrier frequency synchronization and sampling frequency synchronization. It needs FFT units at the transmitter and the receiver. They are sensitive to carrier frequency offset and the spectral efficiency is reduced. To maximize the signal-to- noise ratio and carrierto-interference ratio to achieve a high spectral efficiency and to improve the bit error rate performance we are going for the fast least mean square algorithm.

\subsection{Transmitter Section:}

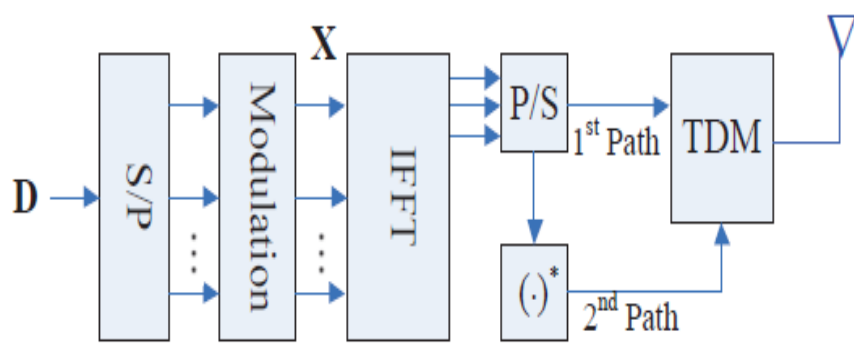

Fig. 1. The transmitter structure of the CC scheme.

Channel Coding and Modulation (BPSK) is that the Channel coding is referred to the processes done in transmitter of a digital communications system. The mentioned processes require allocating extra bits, parity bits, and consuming extra bandwidth. The serial communication is the process of sending data one bit at a time, sequentially, over a communication channel. In parallel communication, the several bits are sent as a whole, on a link with several parallel channels. An IFFT is an algorithm to compute the coefficients of inverse. It converts frequency (or space) to time and vice versa. Next part is the CC scheme can achieve good ICI selfcancellation through two-path conjugate transmission. The transmitter of CC comprises a regular OFDM signal in one path and its conjugate replica in the other path, and these twopath signals can be transmitted using time division multiplexing (TDM). Here the IFFT represents frequency to time so TDM considered.

\subsection{Receiver Section}

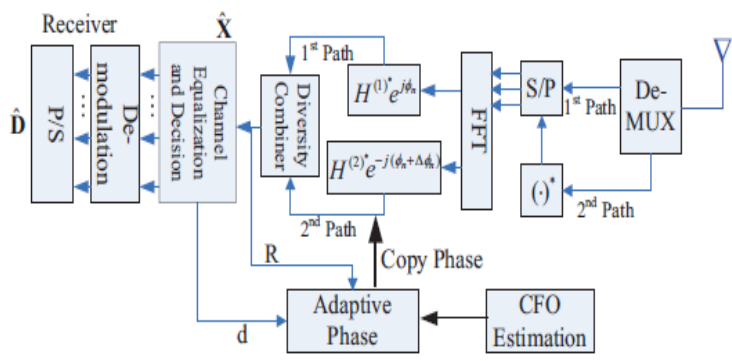

Fig-2: Block diagram of the receiver section.

The receiver in which two artificial phase rotation are employed on both paths. Assuming the OFDM signal passes through an AWGN channel and the CFOs in the two paths are denoted by $\mathrm{H} 1(\varepsilon)$ and $\mathrm{H} 2(\varepsilon+\Delta \varepsilon)$, respectively, we have the following combination output at the receiver after applying two different artificial phase rotations, $\mathrm{H} 1(\varphi)$ and $\mathrm{H} 2(\varphi+\Delta \varphi)$, on the two paths. After completed artificial phase rotation it goes to diversity combiner is the technique applied to combine the multiple received signals of a diversity reception device into a single improved signal. To optimal solution we go for fast least mean square algorithm with channel equalization is used. And finally we get the ICI cancelled signal. In that CIR is maximal.

\section{THE PROPOSED SCHEME:}

The PRCC scheme, in which only one phase rotation is applied at the transmitter, we use two phase rotations on both paths at the proposed receiver to cope with the fast fading channel problems. MMSE equalizer that accounts for the ICI and noise variance can be used to derive a similar adaptive receiver. The MMSE equalizer can further improve the BER performance of the proposed scheme at low to medium SNR it requires additional efforts to estimate the noise variance. The advantage is high spectral efficiency, resistance to frequency selective fading, simple equalization, efficient bandwidth usage, immunity to delay spread.To optimal solutions we go for fast mean square algorithm with channel estimation is used. And finally we get ICI cancelled signal. In that CIR is maximal.

\subsection{The Proposed Receiver Using Two Phase}

\section{Rotations:}

Assume that the OFDM signal passes through the AWGN

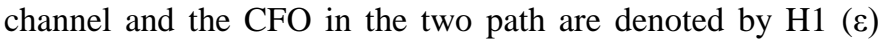
and $\mathrm{H} 2(\varepsilon+\Delta \varepsilon)$, after applying two different artificial phase rotations, $\mathrm{H} 1(\varphi)$ and $\mathrm{H} 2(\varphi+\Delta \varphi)$, on the two paths. CIR $>30 \mathrm{~dB}$ is tolerable under reliable reception. The PRCC with fixed phase rotations cannot always provide the widest tolerable range of CFOs, as compared with the other schemes. The optimal phase rotation in PRCC is that the transmitter 
knows the current $\mathrm{CFO}$ estimation the CIR performance might be sensitive to the CFO estimation error. The performance degradation usually cannot be overcome by directly increasing the feedback rate of the CFO estimate from the receiver to the transmitter during the decrease of the ICI cancellation.

\subsection{Adaptation in Slowly Time-Varying Environments:}

The adaptive receiver is employed with two of the phase rotation where the CFO in two paths as those in fast time varying channels. The channel coherence time is larger than twice of the OFDM symbol duration in the two-path of the transmission.

\subsection{Complexity Analysis:}

The receiver is applied with the MRC for a fair comparison of the three schemes to exploit two-path diversity. The number of complex multiplications is $2 \mathrm{~N}$ for phase rotations. The proposed scheme has a linear increase in computational complexity in contrast to PRCC and CC.

\section{SIMULATION RESULTS:}

In this paper, we propose an adaptive receiver for ICI selfcancellation by taking advantage of conjugate transmission. Unlike the PRCC scheme, in which only one phase rotation is applied at the transmitter, we use two phase rotations on both paths at the proposed receiver to cope with the fast fading channel problems. Under time-varying channels with fast CFO variations the proposed adaptive receiver is superior to $\mathrm{CC}$ and PRCC demonstrated from the computer simulations. The performance improvement provided by the proposed adaptive receiver over $\mathrm{CC}$ and $\mathrm{PRCC}$ is still considerable when the $\mathrm{CFO}$ or channel estimation is taken into account. A minimum mean-squared error (MMSE) equalizer that accounts for the adaptive receiver can be derived from the ICI and noise variance. Although this MMSE equalizer based approach can further improve the BER performance of the proposed scheme at low to medium SNRs, it requires additional efforts to estimate the noise variance.

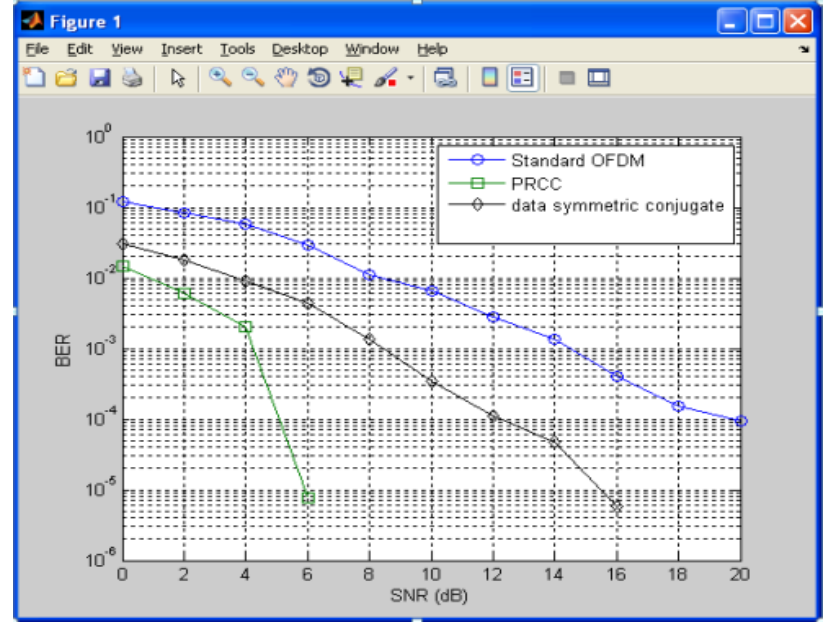

Graph-1: The graph describes BER VS SNR

For adaptive receiver design the receiver how much capable for receiving the samples (bits).The general OFDM, PRCC, symmetric values of conjugation. All describes BER is decreasing and SNR rate achieve high. Depend on SNR value only we can tell, the communication is good or not. Here the SNR is reach high while conjugate cancellation.

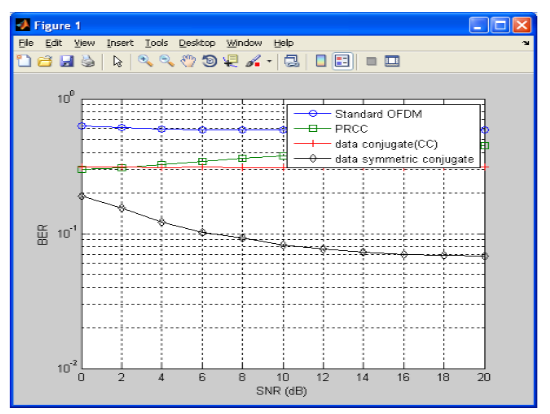

Graph-2: The data conjugate with the offset value.

Graph represents the data conjugate with offset value. Comparing the previous graph the BER values are stabilized in respected SNR value. Data conjugate cancellation process it is improved while applying the offset value.

\section{CONCLUSIONS}

In this paper, we have proposed an efficient of the inter carrier interference (ICI) cancellation in the adaptive receiver using orthogonal frequency division multiplexing system by two path conjugate transmission. The PRCC, which applies one common phase rotation for the two paths at the transmitter, the receiver side uses two artificial phase rotations on the two paths to further address the problems encountered under fast time-varying channels. Besides, the optimal phase rotations 
for maximizing the CIR are derived. By using block least mean-squared algorithm feeding back the CFO information can be eliminated. The proposed adaptive receiver is superior to CC and PRCC, demonstrated by the computer simulation under time-varying channels with fast CFO variations. Compared to a zero-forcing equalizer a minimum meansquared error (MMSE) equalizer that accounts for the ICI and noise variance the proposed adaptive receiver over $\mathrm{CC}$ and PRCC is still considerable. Additional effort is required to estimate the noise variance by the MMSE equalizer to improve the BER performance of the proposed scheme at low to medium SNR. To maximize the carrier-to-interference ratio and signal to noise ratio to achieve a high spectral efficiency by using the fast least mean square algorithm to improve the bit error rate performance. The carrier frequency offset occurs in the mobile environment due to Doppler effects. The CFO occurs due to channel effects the mismatch between the oscillators at the transmitter and the receiver. The CFO problem can be reduced by using the fast least mean square algorithm.To optimal solutions we go for fast mean square algorithm with channel estimation is used. And finally we get ICI cancelled signal. In that CIR is maximal.Here the OFDM is used because it is highly sensitive to the carrier frequency offset.

\section{REFERENCES}

[1]. IEEE TRANSACTION ON COMMUNICATION, VOL.61,NO. 2, FEBRUARY 201359 An Adaptive Receiver Design for OFDM Systems Using Conjugate Transmission.Chin-Liang Wang, Fellow, IEEE, Po-Chung Shen, Student Member, IEEE, Ying-Chang Lin, and Jia-Hong Huang

[2]. R. van Nee and R. Prasad, OFDM for Wireless Multimedia Communications. Artech House, 2000.

[3]. IEEE, "IEEE standard for local and metropolitan area networks-part 11:wireless LAN medium access control (MAC) and physical layer (PHY)specifications," IEEE Std. 802.11, Aug. 1999.

[4]. ETSI, "Digital video broadcasting (DVB): framing structure, channel coding and modulation for digital terrestrial television," ETSI EN 300744 V1.4.1, Jan. 2001.

[5]. IEEE, "IEEE standard for local and metropolitan area networks-part 16:air interface for fixed broadband wireless access systems, Amendment 2: physical and medium access control layers for combined fixed and mobile operation in licensed bands," IEEE Std. 802.16e-2005, Feb. 2006.

[6]. ETSI, "LTE; Evolved Universal Terrestrial Radio Access (E-UTRA);physical channels and modulation (3GPP TS 36.211 version 9.1.0Release 9)," ETSI TS 136211 V9.1.0, Apr. 2010.

[7]. J.-J. van de Beek, M. Sandell, and P. O. Borjesson, "ML estimation of time and frequency offset in OFDM systems," IEEE Trans. Signal Process., vol. 45, no. 7, pp. 1800-1805, July 1997.
[8]. U. Tureli, D. Kivanc, and H. Liu, "Experimental and analytical studies on a high-resolution OFDM carrier frequency offset estimator," IEEE Trans. Veh. Technol., vol. 50, no. 2, pp. 629-643, Mar. 2001.

[9]. Y. Yao and G. B. Giannakis, "Blind carrier frequency offset estimation in SISO, MIMO, and multiuser OFDM systems," IEEE Trans. Commun.,

vol. 53, no. 1, pp. 173-183, Jan. 2005.

[10]. W.-S. Hou and B.-S. Chen, "ICI cancellation for OFDM communication systems in time varying multipath fading channels," IEEE Trans.Wireless Commun., vol. 4, no. 5, pp. 2100-2110, Sep. 2005.

[11]. S. Lu and N. Al-Dhahir, "Coherent and differential ICI cancellation for mobile OFDM with application to DVB-H," IEEE Trans. Wireless Commun., vol. 7, no. 11, pp. 41104116, July 2008.

[12]. Y. Mostofi and D. C. Cox, "ICI mitigation for pilot-aided OFDM mobile systems," IEEE Trans. Wireless Commun., vol. 4, no. 2, pp. 765-774,Mar. 2005.

[13]. Y. Zhao and S.-G. H"aggman, "Intercarrier interference self-cancellation scheme for OFDM mobile communication systems," IEEE Trans. Commun.,vol. 49, no. 7, pp. 11851191, July 2001.

[14]. J. Armstrong, "Analysis of new and existing methods of reducing intercarrier interference due to carrier frequency offset in OFDM," IEEE Trans. Commun., vol. 47, no. 3, pp. 365-369, Mar. 1999.

[15]. K. Sathananthan, C. R. N. Athaudage, and B. Qiu, "A novel ICI cancellation scheme to reduce both frequency offset and IQ imbalance effects in OFDM," in Proc. 2004 IEEE Int. Symp. on Comput. And Commun., pp. 708-713.

[16]. Y. Zhao, J.-D. Leclercq, and S.-G. H"aggman, "Intercarrier interference compression in OFDM communication systems by using correlative coding," IEEE Commun. Lett., vol. 2, pp. 214-216, Aug. 1998.

[17]. C.-L. Wang, Y.-C. Huang, and P.-C. Shen, "An intercarrier interference suppression technique using timedomain windowing," in Proc. 2006 IEEE Veh. Technol. Conf. - Spring, pp. 2518-2522.608 IEEE TRANSACTIONS ON COMMUNICATIONS, VOL. 61, NO. 2, FEBRUARY 2013

[18]. H.-G. Yeh, Y.-K. Chang, and B. Hassibi, "A scheme for cancelling intercarrier interference using conjugate transmission in multicarrier communication systems," IEEE Trans. Wireless Commun., vol. 6, no.1, pp. 3-7, Jan. 2007.

[19]. C.-L. Wang and Y.-C. Huang, "Intercarrier interference cancellation using general phase rotated conjugate transmission for OFDM systems,"IEEE Trans. Commun., vol. 58, no. 3, pp. 812-819, Mar. 2010.

[20]. P. M. Clarkson, Optimal and Adaptive Signal Processing. CRC Press, 1993.

[21]. S. Haykin, Adaptive Filter Theory, 4th edition. PrenticeHall, 2002.

[22]. C.-L. Wang, Y.-C. Lin, and P.-C. Shen, "Design of an adaptive receiver for OFDM systems using conjugate transmission," in Proc. 2010 IEEE Wireless Commun. and Netw. Conf. 
[23]. P. Wan, M. Mcguire, and X. Dong, "Near-optimal channel estimation for OFDM in fast-fading channels," IEEE Trans. Veh. Technol., vol. 60, no. 8, pp. 3780-3791, Oct. 2011.

\section{BIOGRAPHIES}

Chin-Liang Wang (S'85-M'87-SM'04-F'12) was born in Tainan, Taiwan, R.O.C., in 1959. He received the B.S. degree in electronics engineering from National Chiao Tung University (NCTU),Hsinchu, Taiwan, in 1982, the M.S. degree in electrical engineering from National Taiwan University, Taipei, Taiwan, in 1984, and the Ph.D. degree in electronics engineering from NCTU in 1987.He joined the faculty of National Tsing Hua University (NTHU), Hsinchu, Taiwan, in 1987, where he is currently a Professor and Chair of the Department of Electrical Engineering and a Professor of the Institute of Communications. Engineering. During the academic year 1996-1997, he was on leave at the Information Systems Laboratory, Department of Electrical Engineering, Stanford University, Stanford, CA, as a Visiting Scholar. He served as the Director of the Institute of Communications Engineering from 1999 to 2002 and the Director of the Universitys Computer \& Communications Center from 2002 to 2006. He was the Chair of the Wireless Networks Group of the National Science \& Technology Program for Telecommunications from 2004 to 2008, and was the Director of the Communications Engineering Program,National Science Council, R.O.C, from 2009 to 2011. He has been the Chair of the Access Technology Group of the Networked Communications Program since 2009. His current research interests are primarily in baseband technologies for OFDMbased wireless communications, cooperative communications, and cognitive radios.Dr. Wang was a recipient of the NTHU Distinguished Teaching Award in 1992, the Distinguished Teaching Award granted by the Ministry of Education, R.O.C., in 1992, and the Distinguished Electrical Engineering Professor Award granted by the Chinese Institute of Electrical Engineering in 2010. He received the Acer Dragon Thesis Award in 1987 and the Acer Dragon Thesis Advisor Awards in 1995 and 1996. In the academic years 1993-1994 and 19941995, he received the Outstanding Research. Awards from the National Science Council, R.O.C. He received the HDTV Academic Achievement Award from the Digital Video Industry Development Program Office, Ministry of Economic Affairs, R.O.C., in 1996. He was also the advisor on several technical works that won various awards in Taiwan, including the Outstanding Award of the 1993 Texas Instruments DSP Product Design Challenge in Taiwan, the Outstanding Award of the 1994 Contest on Design and Implementation of Microprocessor Application Systems sponsored by the Ministry of Education, R.O.C., the Outstanding Award of the 1995 Student Paper Contest sponsored by the Chinese Institute of Engineers, and the 1995 and 2000 Master Thesis Awards of the Chinese Institute of Electrical Engineering. He served as an Associate Editor for the IEEE TRANSACTIONS ON SIGNAL PROCESSING from 1998 to 2000 and an Editor for Equalization for the IEEE TRANSACTIONSON COMMUNICATIONS from 1998 to 2011. He was also one of the Guest Editors for the Special Issue on Model Order Selection in Signal Processing Systems of the IEEE JOURNAL OF SELECTED TOPICS IN SIGNAL PROCESSING.

Po-Chung Shen (S'06) was born in I-Lan, Taiwan, R.O.C., in 1978. He received the B.S. degree in electrical engineering from National Tsing Hua Universiy(NTHU), Hsinchu, Taiwan, in 2001 and the M.S.degree in communications engineering from NTHU in 2003. He is working toward his Ph.D. degree in communications engineering at NTHU. His research interests include cooperative communications and interference cancellation in OFDM systems.

Ying-Chang Lin was born in Kaohsiung, Taiwan, R.O.C., in 1985. He received the B.S. degree in electrical engineering from National Chi Nan University,Puli, Taiwan, in 2007 and the M.S. degree in communications engineering from National Tsing Hub University, Hsinchu, Taiwan, in 2009. .JiaHung Huang was born in Taipei, Taiwan,R.O.C., in 1986. He received the B.S. degree in electrical engineering from Chang Gung University Taoyuan, Taiwan, in 2008 and the M.S. degree in communications. 